\section{P2-118 VITAMIN C INTAKE FROM DIARY RECORDINGS AND RISK OF BREAST CANCER IN THE UK DIETARY COHORT CONSORTIUM}

${ }^{1} \mathrm{~J}$ Hutchinson, * ${ }^{2} \mathrm{M}$ Lentjes, ${ }^{3} \mathrm{D}$ Greenwood, $\mathrm{V}$ Burley, ${ }^{1} \mathrm{~J}$ Cade, ${ }^{1} \mathrm{C}$ Cleghorn, ${ }^{1} \mathrm{D}$ Threapleton, ${ }^{4} \mathrm{~T}$ Key, ${ }^{4} \mathrm{~B}$ Cairns, ${ }^{5,6} \mathrm{R}$ Keogh, ${ }^{6,7} \mathrm{C}$ Dahm, ${ }^{8} \mathrm{E}$ Brunner, ${ }^{8} \mathrm{M}$ Shipley, ${ }^{9} \mathrm{D}$ Kuh, ${ }^{9} \mathrm{G}$ Mishra, ${ }^{10} \mathrm{~A}$ Stephen, ${ }^{2} \mathrm{~A}$ Bhaniani, ${ }^{2} \mathrm{G}$ Borgulya, ${ }^{2} \mathrm{~K}$ T Khaw, ${ }^{6} \mathrm{~S}$ Rodwell. ${ }^{1}$ Nutritional Epidemiology Group, School of Food Science and Nutrition, University of Leeds, Leeds, UK; ${ }^{2}$ Department of Public Health and Primary Care, University of Cambridge, Cambridge, UK; ${ }^{3}$ Centre of Epidemiology and Biostatistics, University of Leeds, Leeds, UK; ${ }^{4}$ Cancer Epidemiology Unit, University of Oxford, Oxford, UK; ${ }^{5}$ MRC Biostatistics Unit, Institute of Public Health, University of Cambridge, Cambridge, UK; ${ }^{6}$ MRC Centre for Nutritional Epidemiology in Cancer Prevention and Survival, Department of Public Health and Primary Care, University of Cambridge, Cambridge, UK; ' Department of Cardiology, Aarhus University Hospital, Aalborg, Denmark; ${ }^{8}$ Department of Epidemiology and Public Health, University College London, London, UK; ${ }^{9}$ MRC Unit for Lifelong Health and Ageing, Department of Epidemiology and Public Health, University College London, London, UK; ${ }^{10}$ MRC Human Nutrition Research, Elsie Widdowson Laboratory, Cambridge, UK

Introduction Vitamin C intake has been inversely associated with breast cancer risk in case-control studies, but not in meta-analyses of cohort studies using Food Frequency Questionnaires. No study has assessed this relationship prospectively using food diaries which may more accurately measure intake.

Methods Estimated dietary vitamin $\mathrm{C}$ intake was derived from 4 to 7 day food diaries pooled from five prospective studies in the UK Dietary Cohort Consortium. This nested case-control study of 851 incident breast cancer cases and 2727 matched controls examined breast cancer risk in relation to dietary vitamin $\mathrm{C}$ intake using conditional logistic regression adjusting for relevant covariates. Additionally, total vitamin $\mathrm{C}$ intake from supplements and diet was analysed in the three largest cohorts.

Results No evidence of an association was observed between breast cancer risk and dietary $(\mathrm{OR}=1.00$ per $60 \mathrm{mg} / \mathrm{d}, 95 \% \mathrm{CI} 0.91$ to 1.09 , $\mathrm{P}_{\text {trend }}=1.0$ ) or total vitamin C intake (OR=1.01 per $60 \mathrm{mg} / \mathrm{d}, 95 \% \mathrm{CI}$ 1.00 to $\left.1.03, \mathrm{P}_{\text {trend }}=0.1\right)$ in analyses using continuous estimates or by fifths of intake. Additionally, there was no association for postmenopausal women.

Conclusions This pooled analysis of individual UK women found no evidence of associations between breast cancer incidence and dietary or total vitamin $\mathrm{C}$ intake derived uniquely from detailed diary recordings.

\section{P2-119 USE OF SUPPLEMENTS CONTAINING VITAMIN C AND BREAST CANCER RISK IN THE UK WOMEN'S COHORT STUDY}

doi:10.1136/jech.2011.142976i.54 doi:10.1136/jech.2011.142976i.53

Results Compared to women who did not use supplements containing vitamin $\mathrm{C}$, there was no evidence of significant associations between breast cancer incidence and regular vitamin $\mathrm{C}$ supplementation in any intake category. Additionally, no associations were found using continuous estimates ( $\mathrm{HR}=0.98$ per $60 \mathrm{mg} /$ d, $95 \% \mathrm{CI} 0.94$ to $1.02, \mathrm{P}_{\text {trend }}=0.3$ ) or in post-menopausal subanalyses. However pre-menopausal women in the lowest intake category $(\leq 60 \mathrm{mg} / \mathrm{d})$ had significantly increased risks $(\mathrm{HR}=2.37$, 95\% CI 1.32 to 4.27 ) compared to non-users of vitamin C.

Conclusion There was no evidence that supplementation with vitamin C per se was associated with breast cancer incidence in UK women, even at high doses. The increased breast cancer risk found for pre-menopausal women consuming supplements containing vitamin $\mathrm{C}$ less than or equal to $\mathrm{EU}$ recommendations may be due to the effects of other ingredients in these supplements.

\section{P2-120 CONSUMPTION OF COFFEE, TEA, OR GREEN TEA AND ARTERIOLOSCLEROSIS DEVELOPMENT}

doi:10.1136/jech.2011.142976i.55

${ }^{1} \mathrm{~K}$ Ihara, ${ }^{*}{ }^{2} \mathrm{Y}$ Muraoka, ${ }^{3} \mathrm{H}$ lida. ${ }^{1}$ Toho University School of Medicine, Tokyo, Japan, ${ }^{2}$ Kaminoyama Hospital, Yamagata, Japan; ${ }^{3}$ NTT East Japan Izu Hospital, Shizuoka, Japan

Some epidemiological studies have suggested a protective role of beverages such as coffee and tea in stroke, although its relation to arteriolosclerosis has not been examined. We conducted a two-wave prospective study at three work sites to examine the relation of consumption of coffee, tea or green tea to arteriolosclerosis on retina. Both eyes of the participants were photographed with nonmydriatic fundus camera at baseline survey conducted between 1997 and 1999 as well as at follow-up survey conducted between 2002 and 2005. Retinal arteriolosclerotic change judged by a physician with Scheie's classification for both surveys. Beverage consumption of coffee, tea, or green tea was assessed using a selfadministered questionnaire at baseline. Participants at baseline survey were 1587 male aged 43-49, and 1074 were retained at follow-up. Of them, 84 persons with Scheie arteriolosclerosis grade of 2 or more at baseline were excluded from following analysis. At follow-up, 125 persons were newly judged as being with Scheie arteriolosclerosis grade of 2 or more. Those who frequently drank the beverage such as coffee were less likely to develop arteriolosclerosis $(12.0 \%$ for $\geq 3$ cups/day vs $17.7 \%$ for $<1-2$ cups/day). The OR (95\% CI) for developing arteriolosclerosis was 0.64 (0.42 to 0.99) after controlling for possible confounders. Arteriolosclerotic retinal change is strongly correlated with cerebral arteriolosclerosis. Our results suggest that beverages such as coffee, tea, or green tea may have a protective role in sclerotic change of arteriole not only on retina but also in brain.

\section{P2-121 HEAVY METALS EXPOSURE AND BLOOD PRESSURE LEVELS OF RUSSIAN CHILDREN}

doi:10.1136/jech.2011.142976i.56

Introduction Vitamin C supplementation is common in UK women and high doses are often consumed, however evidence is lacking regarding their effects on breast cancer risk in UK users.

Method 11184 middle-aged women from the UK Women's Cohort Study were followed up for a median of 7.4 years. Associations between 239 registered incident breast cancers and vitamin C contained in supplements recorded by 4-day diaries were analysed by Cox's regression models using four intake categories: no frequent use of supplements containing vitamin $\mathrm{C}$; frequent intake up to and including EU recommended allowances ( $\leq 60 \mathrm{mg} / \mathrm{d}$ ); between $60 \mathrm{mg}$ and $500 \mathrm{mg} / \mathrm{d}$; and high intake ( $\geq 500 \mathrm{mg} / \mathrm{d}$ ). Adjustment was made for relevant covariates.
${ }^{1}$ I Ilchenko, ${ }^{*}{ }^{1} \mathrm{M}$ Kotova, ${ }^{1} \mathrm{~K}$ Sharov, ${ }^{2} \mathrm{~S}$ Lyapunov, ${ }^{3}$ I Seregina. ${ }^{1}$ I.M.Sechenov 1-st Moscow State Medical University, Moscow, Russia; ${ }^{2}$ Geological Institute RAS, Moscow, Russia; ${ }^{3}$ M.I.Lomonosov Moscow State University, Moscow, Russia

Introduction Evidence-based data on chronic sub toxic effects of lead, nickel and cerium on blood pressure (BP) of children is insufficient. Methods The target population consisted of a stratified sample of preschool 5-7-year-old children from Gus, 132 children participated (response 80\%) and 211 school children 7-10 y.o. (response 60\%), residing in central district of Moscow. BP was measured in 
accordance with National recommendations for children, 2003. Exposure assessment was based on the levels of scalp hair elements and lead in blood. The analysis was done using ICP-MS and AAS methods. Blood lead samples were analysed using the Lead Care instrument. Multiple logistic regression analysis was done with the adjustment for confounders.

Results Concentrations of studied elements were in subtoxic range, average levels were significantly higher in Gus, then in Moscow. In Gus were revealed strong positive associations of BP, especially diastolic, with the tertile rank of blood Lead (in the range $4.4 \mu \mathrm{g} / \mathrm{dl}$ and higher) and hair cerium (in the range $0.7 \mathrm{mkg} / \mathrm{g}$ and higher). $\mathrm{OR}$ for elevated diastolic BP due to Lead was $3.0 ; 95 \%$ CI 0.59 to 15.76 ; $\mathrm{p}<0.186)$; due to Cerium - 3.9; $95 \%$ CI 1.23 to 12.53 ; $\mathrm{p}<0.021)$. In Moscow BP was significantly correlated with the tertile rank of hair nickel (in the range $0.2 \mathrm{mk} / \mathrm{g}$ and higher). OR for elevated systolic $\mathrm{BP}$ due to nickel was 2.5 (95\% CI 1.1 to 5.7, $\mathrm{p}<0.026)$; for diastolic BP - 5,6; 95\% CI 2.2 to 14.6; $\mathrm{p}<0.001)$.

Conclusions The blood Lead, hair Nickel and Cerium levels, even in the low range of concentrations, positively associates with the risk of elevated blood pressure in children.

\section{P2-122 RESPONSE AND PREDICTORS OF RESPONSE, TO PEGYLATED INTERFERON AND RIBAVIRIN FOR CHRONIC HEPATITIS C PATIENTS IN SCOTLAND: ALANINE AMINOTRANSFERASE (ALT) AND GAMMA GLUTAMYL TRANSFERASE (GGT) ARE VALUABLE PRE-TREATMENT MARKERS OF AN SVR IN ROUTINE CLINICAL PRACTICE}

doi:10.1136/jech.2011.142976i.57

\begin{abstract}
1,2 H Innes, ${ }^{* 1,2} \mathrm{~S}$ Hutchinson, ${ }^{3} \mathrm{~S}$ Allen, ${ }^{4} \mathrm{D}$ Bhattacharyya, ${ }^{5} \mathrm{P}$ Bramley, ${ }^{6} \mathrm{~B}$ Carman, ${ }^{7,8} \mathrm{~T}$ Delahooke, ${ }^{9} \mathrm{~J}$ Dillon, ${ }^{1} \mathrm{D}$ Goldberg, ${ }^{10} \mathrm{~N}$ Kennedy, ${ }^{11} \mathrm{P}$ Mills, ${ }^{12} \mathrm{~J}$ Morris, ${ }^{13} \mathrm{~J}$ Morris, ${ }^{12} \mathrm{~A}$ Stanley, ${ }^{1} \mathrm{P}$ Hayes. ${ }^{1}$ Health Protection Scotland, Glasgow, UK; ${ }^{2}$ Department of Mathematics and Statistics, University of Strathclyde, Glasgow, UK; ${ }^{3}$ Crosshouse Hospital, Kilmarnock, UK; ${ }^{4}$ Victoria Hospital, Kirkcaldy, UK; ${ }^{5}$ Stirling Royal Infirmary, Stirling, UK; ${ }^{6}$ West of Scotland Specialist Virology Centre, Glasgow, UK; ${ }^{7}$ Royal Infirmary Edinburgh, Edinburgh, UK; ${ }^{8}$ Leicester Royal Infirmary, Leicester, UK; ${ }^{9}$ Ninewells Hospital and medical school, Dundee, UK; ${ }^{10}$ Monklands Hospital, Airdrie, UK; ${ }^{11}$ Gartnavel General Hospital, Glasgow, UK; ${ }^{12}$ Glasgow Royal Infirmary, Glasgow, UK; ${ }^{13}$ Southern General Hospital, Glasgow, UK
\end{abstract}

Introduction It is not clear what proportion of HCV (hepatitis C virus) patients attain a sustained viral response (SVR) when treated with pegylated interferon and ribavirin combination therapy outside randomised clinical trials (RCTs). Secondly, pre-treatment factors available in routine clinical settings that are predictive of SVR (the optimal treatment outcome) are not known.

Methods HCV clinical databases from nine Scottish treatment clinics were used to derive a retrospective cohort of 934 patients initiated on HCV treatment during 2000-2007.

Results In our cohort, 39\% $(123 / 315$, 95\% CI $34 \%$ to $45 \%)$ of genotype (GT) 1, and 70\% (414/594, 95\% CI $66 \%$ to $73 \%$ ) of genotype $2 / 3$ (GT2/3) patients achieved a SVR; this compares with pooled estimates of $47 \%$ for GT1 ( $95 \%$ CI $41 \%$ to $52 \%$ ), and $80 \%$ for GT2/3 (95\% CI 75\% to 85\%) patients from RCTs. Pre-treatment factors significantly associated with SVR were: gamma glutamyl transferase (GGT) $\geq 55 \mathrm{IU} / 1$ (adjusted OR: $0.46,95 \%$ CI 0.33 to $0.65)$, platelet count $\geq 150 \times 10^{9} / 1(1.92,95 \%$ CI 1.26 to 2.93$)$, ALT quotient $\geq 2.5$ (for those GT1 infected: $2.66,95 \%$ CI 1.46 to 4.84 ), GT2/3 (for those with ALT quotient <2.5: 4.05, 95\% CI 2.82 to 5.80; and for those with ALT quotient $\geq 2.5$ : $1.91,95 \%$ CI 1.01 to 3.61 ), age (per ten year increase) $(0.84,95 \%$ CI 0.72 to 0.99$)$, ever HBV infection $(0.67,95 \% \mathrm{CI} 0.45$ to 0.98$)$, and male gender $(0.70,95 \% \mathrm{CI}$ 0.50 to 0.98$)$.

Conclusions The principal conclusions are twofold: (1) the proportion of patients attaining a SVR in Scottish routine practice is marginally lower than in RCTs, and (2) in addition to genotype,
GGT (in all patients) and ALT (in GT1 patients only) emerge as valuable predictors of an SVR in the routine clinical setting.

\section{P2-123 EVALUATION OF RISK FACTOR AND PROTECTION FOR CHRONIC NON COMMUNICABLE DISEASES MONITORING SYSTEM BY PHONE SURVEY: VIGITEL, BRAZIL 2006 TO 2008}

doi:10.1136/jech.2011.142976i.58

${ }^{1} \mathrm{~B}$ Iser, ${ }^{*}{ }^{2} \mathrm{E}$ Macário, ${ }^{1} \mathrm{~J}$ Sobel, ${ }^{1} \mathrm{~L}$ Moura, ${ }^{1} \mathrm{D}$ Malta. ${ }^{1}$ National Coordination of Injury and Non-Communicable Disease Surveillance, Department of Health Analysis, Secretariat of Health Surveillance (SVS), Ministry of Health (MoH), Brasilia, Distrito Federal, Brazil; ${ }^{2}$ Field Epidemiology Training Program, Ministry of Health, Brasilia, Distrito Federal , Brazil; ${ }^{3}$ Center for Global Health, CDC, Altlanta, Georgia, USA

Vigitel is a system that monitors risk factors by means of continuous telephone interviews on samples of adults living in households with landline telephones in the Brazilian capital. To evaluate the system from 2006 to 2008, we used the Updated Guidelines for Evaluating Public Health Surveillance Systems from CDC, semistructured questionnaires for interviews with key-informants and comparisons to other surveys, such as the BRFSS.

Results The Vigitel operation has seven steps: sample selection, eligible households identification, individual selection, interview, check, closure, data management. The number of questions ranged from 78 to 92 . Among state coordinators, $96 \%$ demonstrated good knowledge of objectives and $86 \%$ of outcomes and among interviewers, $54 \%$ to $36 \%$, respectively, indicating acceptability. Lack of operation process documentation proved stability. Completeness of variables was greater than $90 \%$ and response rate higher than $70 \%$. Prevalence of smoking, high blood pressure and obesity were similar to other surveys, indicating the sensitivity to capture these factors. Changes in workers, in the questionnaire and the software didn't affect the operation. Time between final data collection and data availability was 3 months. Telephone company coverage of $80 \%$ probability sampling and data weighting make it representative. The system allows use of indicators in planning of interventions and policy development.

Conclusions The system demonstrated simplicity, acceptability, stability, and good data quality. It was flexible, opportune, representative and useful in monitoring risk factors in population. Improvement in dissemination of results, allowing local workers to perform data analysis and document system operation is necessary for to improve the system.

\section{P2-124 LOWER LIPOPROTEIN(A) LEVELS CAUSE CEREBRAL HAEMORRHAGE: THE JICHI MEDICAL SCHOOL COHORT STUDY}

doi:10.1136/jech.2011.142976i.59

${ }^{1} \mathrm{~S}$ Ishikawa, ${ }^{2} \mathrm{~K}$ Kayaba, ${ }^{1} \mathrm{~T}$ Gotoh, ${ }^{1} \mathrm{Y}$ Nakamura, ${ }^{1} \mathrm{E}$ Kajii. ${ }^{1}$ Jichi Medical University, Tochigi, Japan; ${ }^{2}$ Saitama Prefectural University, Saitama, Japan

Introduction Lipoprotein(a) ( $\operatorname{Lp}(\mathrm{a}))$ plays an important role in atherosclerosis. Some observational studies report $\mathrm{Lp}(\mathrm{a})$ as positively associated with myocardial infarction, but the relationship between $\mathrm{Lp}(\mathrm{a})$ and stroke is unclear. We examined the relationship between $\mathrm{Lp}(\mathrm{a})$ and the incidence of stroke in the general population.

Methods A multi-center population-based cohort study was conducted. A total of 10444 men and women were eligible. Data were obtained from April 1992 to July 1995 in 12 rural districts in Japan. Subjects were divided into tertiles of $L p(a)$ levels. We analysed the risks of all stroke and of stroke subtypes in each sex using Cox's proportional hazard models. 